UDC: $517.57: 517.16$

\title{
SOME NEW TYPE INTEGRAL INEQUALITIES FOR APPROXIMATELY HARMONIC $h$-PREINVEX FUNCTIONS
}

\author{
BADREDDINE MEFTAH ${ }^{1}$ AND ARTION KASHURI ${ }^{2}$
}

\begin{abstract}
In this paper, we present a new definition of preinvex functions depending on the given function which is called as approximately harmonic $h$-preinvex functions. We establish some inequalities of Hermite-Hadamard, Simpson and midpoint type for newly define class of functions. We show that this class contains several other new and known extensions of harmonic preinvexity. Special cases which can be deduced from the main results are also discussed in detail.
\end{abstract}

\section{Introduction and Preliminaries}

The Hermite-Hadamard inequalities are the most well established inequalities in the theory of convex functions with a geometrical interpretation and many applications. These inequalities state that, if $f: I \rightarrow \mathbb{R}$ is a convex function on the interval $I$ of real numbers and $a, b \in I$ with $a<b$, then

$$
f\left(\frac{a+b}{2}\right) \leq \frac{1}{b-a} \int_{a}^{b} f(x) d x \leq \frac{f(a)+f(b)}{2} .
$$

Both inequalities in (1.1) hold in the reversed direction if $f$ is concave. We note that Hermite-Hadamard inequality may be regarded as a refinement of the concept of convexity and it follows easily from Jensen's inequality. Hermite-Hadamard inequality for convex functions has received renewed attention in recent years and a remarkable variety of refinements and generalizations have been studied, see $[2,4,5,6,17,8,11,12,13,15,16,17,18,30$.

Let us recall now some basic definitions that will be useful in our paper.

Definition 1.1. [29] A set $I \subset \mathbb{R} \backslash\{0\}$ is said to be harmonically convex, if

$$
\frac{x y}{t x+(1-t) y} \in K
$$

2010 Mathematics Subject Classification. Primary: 26A51, 26B25, 26D10, 26D15.

Key words and phrases. Hermite-Hadamard inequalities, Simpson inequality, midpoint inequality, Hölder inequality, power mean inequality, harmonically preinvex. 
holds for all $x, y \in K$ and $t \in[0,1]$.

Definition 1.2. 9] A function $f:[a, b] \subset \mathbb{R} \backslash\{0\} \rightarrow \mathbb{R}$ is said to be harmonic convex function, if

$$
f\left(\frac{x y}{t x+(1-t) y}\right) \leq(1-t) f(x)+t f(y)
$$

holds for all $x, y \in[a, b]$ and $t \in[0,1]$.

Definition 1.3. 20] A function $f:[a, b] \subset \mathbb{R} \backslash\{0\} \rightarrow \mathbb{R}$ is said to be harmonic P-function, if

$$
f\left(\frac{x y}{t x+(1-t) y}\right) \leq f(x)+f(y)
$$

holds for all $x, y \in[a, b]$ and $t \in[0,1]$.

Definition 1.4. 20] A function $f:[a, b] \subset \mathbb{R}_{+} \backslash\{0\} \rightarrow \mathbb{R}$ is said to be harmonic $s$-convex in the second sense for some fixed $s \in(0,1]$, if

$$
f\left(\frac{x y}{y x+(1-t) y}\right) \leq(1-t)^{s} f(x)+t^{s} f(y)
$$

holds for all $x, y \in[a, b]$ and $t \in[0,1]$.

Definition 1.5. 20] A function $f:[a, b] \subset \mathbb{R} \backslash\{0\} \rightarrow \mathbb{R}$ is said to be harmonic Godunova-Levin function with respect to $\eta$, if

$$
f\left(\frac{x y}{t x+(1-t) y}\right) \leq(1-t)^{-1} f(x)+t^{-1} f(y)
$$

holds for all $x, y \in[a, b]$ and $t \in(0,1)$.

Definition 1.6. 20] A function $f:[a, b] \subset \mathbb{R} \backslash\{0\} \rightarrow \mathbb{R}$ is said to be harmonic $s$-Godunova-Levin function with respect to $\eta$, if

$$
f\left(\frac{x y}{t x+(1-t) y}\right) \leq(1-t)^{-s} f(x)+t^{-s} f(y)
$$

holds for all $x, y \in[a, b]$ and $t \in(0,1)$.

Definition 1.7. 25]. A function $f:[a, b] \subset \mathbb{R} \backslash\{0\} \rightarrow \mathbb{R}$ is said to be harmonic tgs-convex function, if

$$
f\left(\frac{x y}{t x+(1-t) y}\right) \leq t(1-t)(f(x)+f(y))
$$

holds for all $x, y \in[a, b]$, and $t \in(0,1)$.

Definition 1.8. [23] A function $f:[a, b] \subset \mathbb{R} \backslash\{0\} \rightarrow \mathbb{R}$ is said to be harmonic MT-convex function, if

$$
f\left(\frac{x y}{t x+(1-t) y}\right) \leq \frac{\sqrt{1-t}}{2 \sqrt{t}} f(x)+\frac{\sqrt{t}}{2 \sqrt{1-t}} f(y)
$$

holds for all $x, y \in[a, b]$ and $t \in(0,1)$. 
Definition 1.9. [3] A function $f:[a, b] \subset \mathbb{R} \backslash\{0\} \rightarrow \mathbb{R}$ is said to be harmonic beta-convex function, if

$$
f\left(\frac{x y}{t x+(1-t) y}\right) \leq t^{q}(1-t)^{p} f(x)+t^{p}(1-t)^{q} f(y)
$$

holds for all $x, y \in[a, b], p, q>-1$ and $t \in(0,1)$.

Definition 1.10. 20] A function $f:[a, b] \subset \mathbb{R} \backslash\{0\} \rightarrow \mathbb{R}$ is said to be harmonic $h$-convex function, if

$$
f\left(\frac{x y}{t x+(1-t) y}\right) \leq h(1-t) f(x)+h(t) f(y)
$$

holds for all $x, y \in[a, b]$ and $t \in[0,1]$.

Very recently, Awan et al. [1, introduced the following new class called approximately harmonic $h$-convex function.

Definition 1.11. [1] A function $f: K \rightarrow \mathbb{R}_{+}$is said to be approximately harmonic h-convex function, if

$$
f\left(\frac{x y}{t x+(1-t) y}\right) \leq h(1-t) f(x)+h(t) f(y)+d(x, y)
$$

holds for all $x, y \in K$ and $t \in(0,1)$.

The above definition recapture all definition cited for $d(x, y)=0$, strongly harmonic $h$-convexity, see [25], $\gamma$-paraharmonic $h$-convexity, $\epsilon$-paraharmonic $h$-convexity and relaxed harmonic $h$-convexity and their subclasses, that is to say convex, $s$-convex, $P$-function, $Q$-functions, $s$ - $Q$-functions, $M T$-convex, tgs-convex and beta-convex, see [1].

Definition 1.12. 22] A set $K \subset \mathbb{R} \backslash\{0\}$ is said to be harmonically preinvex, if

$$
\frac{x(x+\eta(y, x))}{x+(1-t) \eta(y, x)} \in K
$$

holds for all $x, y \in K$ and $t \in[0,1]$.

Definition 1.13. 24] A function $f:[a, a+\eta(b, a)] \subset \mathbb{R} \backslash\{0\} \rightarrow \mathbb{R}$ is said to be harmonic preinvex function with respect to $\eta$, if

$$
f\left(\frac{x(x+\eta(y, x))}{x+(1-t) \eta(y, x)}\right) \leq(1-t) f(x)+t f(y)
$$

holds for all $x, y \in[a, a+\eta(b, a)]$ and $t \in[0,1]$.

Definition 1.14. 24] A function $f:[a, a+\eta(b, a)] \subset \mathbb{R} \backslash\{0\} \rightarrow \mathbb{R}$ is said to be harmonic P-preinvex function with respect to $\eta$, if

$$
f\left(\frac{x(x+\eta(y, x))}{x+(1-t) \eta(y, x)}\right) \leq f(x)+f(y)
$$

holds for all $x, y \in[a, a+\eta(b, a)]$ and $t \in[0,1]$. 
Definition 1.15. 24] A function $f:[a, a+\eta(b, a)] \subset \mathbb{R} \backslash\{0\} \rightarrow \mathbb{R}$ is said to be harmonic s-preinvex function in the second sense with respect to $\eta$ for some fixed $s \in(0,1]$, if

$$
f\left(\frac{x(x+\eta(y, x))}{x+(1-t) \eta(y, x)}\right) \leq(1-t)^{s} f(x)+t^{s} f(y)
$$

holds for all $x, y \in[a, a+\eta(b, a)]$ and $t \in[0,1]$.

Definition 1.16. [24] A function $f:[a, a+\eta(b, a)] \subset \mathbb{R} \backslash\{0\} \rightarrow \mathbb{R}$ is said to be harmonic Godunova-Levin preinvex function with respect to $\eta$, if

$$
f\left(\frac{x(x+\eta(y, x))}{x+(1-t) \eta(y, x)}\right) \leq \frac{f(x)}{1-t}+\frac{f(y)}{t}
$$

holds for all $x, y \in[a, a+\eta(b, a)]$ and $t \in(0,1)$.

Definition 1.17. 24] A function $f:[a, a+\eta(b, a)] \subset \mathbb{R} \backslash\{0\} \rightarrow \mathbb{R}$ is said to be harmonic s-Godunova-Levin preinvex function with respect to $\eta$, if

$$
f\left(\frac{x(x+\eta(y, x))}{x+(1-t) \eta(y, x)}\right) \leq \frac{f(x)}{(1-t)^{s}}+\frac{f(y)}{t^{s}}
$$

holds for all $x, y \in[a, a+\eta(b, a)]$ and $t \in(0,1)$.

Definition 1.18. [28, A function $f:[a, a+\eta(b, a)] \subset \mathbb{R} \backslash\{0\} \rightarrow \mathbb{R}$ is said to be harmonic tgs-preinvex function with respect to $\eta$, if

$$
f\left(\frac{x(x+\eta(y, x))}{x+(1-t) \eta(y, x)}\right) \leq t(1-t)(f(x)+f(y))
$$

holds for all $x, y \in[a, a+\eta(b, a)]$ and $t \in(0,1)$.

Definition 1.19. 27] A function $f:[a, a+\eta(b, a)] \subset \mathbb{R} \backslash\{0\} \rightarrow \mathbb{R}$ is said to be harmonic MT-preinvex function with respect to $\eta$, if

$$
f\left(\frac{x(x+\eta(y, x))}{x+(1-t) \eta(y, x)}\right) \leq \frac{\sqrt{1-t}}{2 \sqrt{t}} f(x)+\frac{\sqrt{t}}{2 \sqrt{1-t}} f(y)
$$

holds for all $x, y \in[a, a+\eta(b, a)]$ and $t \in(0,1)$.

Definition 1.20. [28] A function $f:[a, a+\eta(b, a)] \subset \mathbb{R} \backslash\{0\} \rightarrow \mathbb{R}$ is said to be harmonic beta-preinvex function with respect to $\eta$, if

$$
f\left(\frac{x(x+\eta(y, x))}{x+(1-t) \eta(y, x)}\right) \leq t^{q}(1-t)^{p} f(x)+t^{p}(1-t)^{q} f(y)
$$

holds for all $x, y \in[a, a+\eta(b, a)], p, q>-1$ and $t \in(0,1)$.

Definition 1.21. 24] A function $f:[a, a+\eta(b, a)] \subset \mathbb{R} \backslash\{0\} \rightarrow \mathbb{R}$ is said to be harmonic h-preinvex function with respect to $\eta$, if

$$
f\left(\frac{x(x+\eta(y, x))}{x+(1-t) \eta(y, x)}\right) \leq h(1-t) f(x)+h(t) f(y)
$$

holds for all $x, y \in[a, a+\eta(b, a)]$ and $t \in[0,1]$. 
Condition $\mathbf{C}[19$ Let $I \subset \mathbb{R}$ be an invex set with respect to $\eta$, for any $x, y \in I$ and any $t \in[0,1]$, we have

$$
\begin{aligned}
& \eta(y, y+t \eta(x, y))=-t \eta(x, y) \\
& \eta(x, y+t \eta(x, y))=(1-t) \eta(x, y) .
\end{aligned}
$$

Note that for every $x, y \in I$ and any $t_{1}, t_{2} \in[0,1]$ and from Condition C, we have

$$
\eta\left(y+t_{2} \eta(x, y), y+t_{1} \eta(x, y)\right)=\left(t_{2}-t_{1}\right) \eta(x, y) .
$$

We also recall some useful special functions, see [14].

The beta function is defined as

$$
B(x, y)=\int_{0}^{1} t^{x-1}(1-t)^{y-1}=\frac{\Gamma(x) \Gamma(y)}{\Gamma(x+y)} .
$$

The hypergeometric function is defined by

$$
{ }_{2} F_{1}(a, b, c, z)=\frac{1}{B(b, c-b)} \int_{0}^{1} t^{b-1}(1-t)^{c-b-1}(1-z t)^{-a} d t,
$$

for $c>b>0$ and $|z|<1$.

Motivated by the above literature, the main objective of this paper is to establish some inequalities of Hermite-Hadamard, Simpson and midpoint type for a new definition of preinvex functions depending on the given function, which will be called approximately harmonic $h$-preinvex functions. We will show that this class contains several other new and known extensions of harmonic preinvexity. Special cases which will be deduced from the main results will be discussed in detail. At the end, a brief conclusion will be provided as well.

\section{Main Results}

In what follows $(X,\|\|$.$) be a real normed space, d: X \times X \rightarrow \mathbb{R}$ and $h:(0,1) \rightarrow \mathbb{R}$, $K \subset X$ be harmonically preinvex set such that $[a, a+\eta(b, a)] \subset K \subseteq \mathbb{R} \backslash\{0\}$ with $\eta(b, a)>0$.

We are in position to introduce a new interesting definition of preinvex functions depending on the given function, which will be called approximately harmonic $h$ preinvex function, as follows:

Definition 2.1. A function $f: K \rightarrow \mathbb{R}_{+}$is said to be approximately harmonic h-preinvex function, if

$$
f\left(\frac{x(x+\eta(y, x))}{x+t \eta(y, x)}\right) \leq h(1-t) f(x)+h(t) f(y)+d(x, x+\eta(y, x))
$$

holds for all $x, y \in K$ and $t \in(0,1)$.

From our Definition 2.1 follows new interesting definitions: 
Definition 2.2. A function $f: K \rightarrow \mathbb{R}_{+}$is said to be $\gamma$-paraharmonic $h$-preinvex function, if

$$
f\left(\frac{x(x+\eta(y, x))}{x+t \eta(y, x)}\right) \leq h(1-t) f(x)+h(t) f(y)+\varepsilon|\eta(y, x)|^{\delta} .
$$

holds for all $x, y \in K$ and $t \in(0,1)$.

Definition 2.3. A function $f: K \rightarrow \mathbb{R}_{+}$is said to be $\epsilon$-paraharmonic h-preinvex function, if

$$
f\left(\frac{x(x+\eta(y, x))}{x+t \eta(y, x)}\right) \leq h(1-t) f(x)+h(t) f(y)+\epsilon|\eta(y, x)|
$$

holds for all $x, y \in K$ and $t \in(0,1)$.

Definition 2.4. A function $f: K \rightarrow \mathbb{R}_{+}$is said to be strongly harmonic h-preinvex function, if

$$
f\left(\frac{x(x+\eta(y, x))}{x+t \eta(y, x)}\right) \leq h(1-t) f(x)+h(t) f(y)-c t(1-t)\left(\frac{1}{x+\eta(y, x)}-\frac{1}{x}\right)^{2}
$$

holds for all $x, y \in K$ and $t \in(0,1)$.

Definition 2.5. A function $f: K \rightarrow \mathbb{R}_{+}$is said to be relaxed harmonic h-preinvex function, if

$f\left(\frac{x(x+\eta(y, x))}{x+t \eta(y, x)}\right) \leq h(1-t) f(x)+h(t) f(y)+c t(1-t)\left(\frac{1}{x+\eta(y, x)}-\frac{1}{x}\right)^{2}$

holds for all $x, y \in K$ and $t \in(0,1)$.

Theorem 1. Let $f:[a, a+\eta(b, a)] \subseteq \mathbb{R} \backslash\{0\} \rightarrow \mathbb{R}$ be an integrable function. If $f$ is approximately harmonic h-preinvex function, and Condition $C$ holds, then

$$
\begin{gathered}
\frac{1}{2 h\left(\frac{1}{2}\right)}\left(f\left(\frac{2 a(a+\eta(b, a))}{2 a+\eta(b, a)}\right)-\frac{a(a+\eta(b, a))}{\eta(b, a)} \int_{a}^{a+\eta(b, a)} \frac{d\left(u,\left(\frac{1}{a}+\frac{1}{a+\eta(b, a)}-\frac{1}{u}\right)^{-1}\right)}{u^{2}} d u\right) \leq \\
\leq \frac{a(a+\eta(b, a))}{\eta(b, a)} \int_{a}^{a+\eta(b, a)} \frac{f(x)}{x^{2}} d x \leq \\
\leq(f(a)+f(b)) \int_{0}^{1} h(t) d t+\int_{0}^{1} d(a, a+\eta(b, a)) d t .
\end{gathered}
$$

Proof. It follows from the approximately harmonic $h$-preinvexity of $f$ that

$$
f\left(\frac{2 x(x+\eta(y, x))}{2 x+\eta(y, x)}\right) \leq h\left(\frac{1}{2}\right)(f(x)+f(y))+d(x, x+\eta(y, x)) .
$$

Let $x=\frac{a(a+\eta(b, a))}{a+t \eta(b, a)}$ and $y=\frac{a(a+\eta(b, a))}{a+(1-t) \eta(b, a)}$. Using Condition C, we get

$$
f\left(\frac{2 a(a+\eta(b, a))}{2 a+\eta(b, a)}\right) \leq
$$




$$
\begin{aligned}
& \leq h\left(\frac{1}{2}\right)\left(f\left(\frac{a(a+\eta(b, a))}{a+t \eta(b, a)}\right)+f\left(\frac{a(a+\eta(b, a))}{a+(1-t) \eta(b, a)}\right)\right)+ \\
& +d\left(\frac{a(a+\eta(b, a))}{a+\operatorname{t\eta }(b, a)}, \frac{a(a+\eta(b, a))}{a+(1-t) \eta(b, a)}\right) .
\end{aligned}
$$

Integrating both sides of $(2.1)$ with respect to $t$ over $[0,1]$, we obtain

$$
\begin{aligned}
& f\left(\frac{2 a(a+\eta(b, a))}{2 a+\eta(b, a)}\right) \\
\leq & h\left(\frac{1}{2}\right)\left(\int_{0}^{1} f\left(\frac{a(a+\eta(b, a))}{a+t \eta(b, a)}\right) d t+\int_{0}^{1} f\left(\frac{a(a+\eta(b, a))}{a+(1-t) \eta(b, a)}\right) d t\right) \\
+ & \int_{0}^{1} d\left(\frac{a(a+\eta(b, a))}{a+t \eta(b, a)}, \frac{a(a+\eta(b, a))}{a+(1-t) \eta(b, a)}\right) d t \\
= & h\left(\frac{1}{2}\right)\left(2 \frac{a(a+\eta(b, a))}{\eta(b, a)} \int_{a}^{a+\eta(b, a)} \frac{f(u)}{u^{2}} d u\right) \\
+ & \frac{a(a+\eta(b, a))}{\eta(b, a)} \int_{a}^{a+\eta(b, a)} d\left(u,\left(\frac{1}{a}+\frac{1}{a+\eta(b, a)}-u\right)^{-1}\right) \frac{d u}{u^{2}} .
\end{aligned}
$$

From 2.2 , we have

$$
\begin{gathered}
\frac{1}{2 h\left(\frac{1}{2}\right)}\left(f\left(\frac{2 a(a+\eta(b, a))}{2 a+\eta(b, a)}\right)-\frac{a(a+\eta(b, a))}{\eta(b, a)} \frac{d\left(u,\left(\frac{1}{a}+\frac{1}{a+\eta(b, a)}-u\right)^{-1}\right)}{u^{2}} d u\right) \leq \\
\leq \frac{a(a+\eta(b, a))}{\eta(b, a)} \int_{a}^{a+\eta(b, a)} \frac{f(u)}{u^{2}} d u
\end{gathered}
$$

On the other hand

$$
f\left(\frac{a(a+\eta(b, a))}{a+t \eta(b, a)}\right) \leq h(1-t) f(a)+h(t) f(b)+d(a, a+\eta(b, a)) .
$$

Integrating both sides of $(2.4)$ with respect to $t$ over $[0,1]$, we get

$$
\int_{0}^{1} f\left(\frac{a(a+\eta(b, a))}{a+t \eta(b, a)}\right) \leq \int_{0}^{1}\{h(1-t) f(a)+h(t) f(b)+d(a, a+\eta(b, a))\} d t
$$


which implies that

$\frac{a(a+\eta(b, a))}{\eta(b, a)} \int_{a}^{a+\eta(b, a)} \frac{f(u)}{u^{2}} d u \leq(f(a)+f(b)) \int_{0}^{1} h(t) d t+\int_{0}^{1} d(a, a+\eta(b, a)) d t$.

The desired result follows from 2.3$)$ and $(2.5)$. The proof is completed.

Remark 2.1: (1) Theorem 1 will be reduced to Theorem 3.1 from [1], if we choose $\eta(b, a)=b-a$.

(2) Theorem 1 will be reduced to Theorem 2.4 from [21, if $d(a, a+\eta(b, a))=0$. Moreover, if we choose $\eta(b, a)=b-a$, we recapture Theorem 3.2 from [20].

Corollary 1.1. Let $f:[a, a+\eta(b, a)] \subseteq \mathbb{R} \backslash\{0\} \rightarrow \mathbb{R}$ be an integrable function. If $f$ is approximately harmonic preinvex function, and Condition $C$ holds, then

$$
\begin{gathered}
f\left(\frac{2 a(a+\eta(b, a))}{2 a+\eta(b, a)}\right)-\frac{a(a+\eta(b, a))}{\eta(b, a)} \int_{a}^{a+\eta(b, a)} \frac{d\left(u,\left(\frac{1}{a}+\frac{1}{a+\eta(b, a)}-\frac{1}{u}\right)^{-1}\right)}{u^{2}} d u \\
\leq \frac{a(a+\eta(b, a))}{\eta(b, a)} \int_{a}^{a+\eta(b, a)} \frac{f(x)}{x^{2}} d x \leq \frac{f(a)+f(b)}{2}+\int_{0}^{1} d(a, a+\eta(b, a)) d t .
\end{gathered}
$$

Remark 2.2: (1) Corollary 1.1 will be reduced to Corollary 3.2 from [1], if we take $\eta(b, a)=b-a$. Moreover, we obtain Theorem 2.2 from [26], if we choose $d(a, a+\eta(b, a))=-c t(1-t)\left(\frac{1}{a+\eta(b, a)}-\frac{1}{a}\right)^{2}$.

(2) Corollary 1.1 will be reduced to Theorem 2.1 from $[22$, if $d(a, a+\eta(b, a))=$ 0 . Moreover, if we choose $\eta(b, a)=b-a$, we get Theorem 2.4 from [9].

Corollary 1.2. Let $f:[a, a+\eta(b, a)] \subseteq \mathbb{R} \backslash\{0\} \rightarrow \mathbb{R}$ be an integrable function. If $f$ is approximately harmonic P-preinvex function, and Condition $C$ holds, then

$$
\begin{aligned}
& \frac{1}{2} f\left(\frac{2 a(a+\eta(b, a))}{2 a+\eta(b, a)}\right)-\frac{a(a+\eta(b, a))}{2 \eta(b, a)} . \\
& \cdot \int_{a}^{a+\eta(b, a)} \frac{d\left(u,\left(\frac{1}{a}+\frac{1}{a+\eta(b, a)}-\frac{1}{u}\right)^{-1}\right)}{u^{2}} d u \\
& \leq \frac{a(a+\eta(b, a))}{\eta(b, a)} \int_{a}^{a+\eta(b, a)} \frac{f(x)}{x^{2}} d x \leq f(a)+f(b)+\int_{0}^{1} d(a, a+\eta(b, a)) d t .
\end{aligned}
$$

Remark 2.3: (1) Taking $\eta(b, a)=b-a$, in Corollary 1.2 we obtain Corollary 3.5 from [1]. Note that in the inequality on the left it is missing $\frac{1}{2}$. 
(2) Corollary 1.2 will be reduced to Corollary 2.4 from [21, if $d(a, a+\eta(b, a))=$ 0 . Moreover, if we choose $\eta(b, a)=b-a$, we obtain Corollary 3.4 from [20].

Corollary 1.3. Let $f:[a, a+\eta(b, a)] \subseteq \mathbb{R} \backslash\{0\} \rightarrow \mathbb{R}$ be an integrable function. If $f$ is approximately harmonic Godunova-Levin-Dragomir-preinvex function, and Condition C holds, then

$$
\begin{array}{r}
2^{-(1+s)}\left(f\left(\frac{2 a(a+\eta(b, a))}{2 a+\eta(b, a)}\right)-\right. \\
\left.-\frac{a(a+\eta(b, a))}{\eta(b, a)} \int_{a}^{a+\eta(b, a)} \frac{d\left(u,\left(\frac{1}{a}+\frac{1}{a+\eta(b, a)}-\frac{1}{u}\right)^{-1}\right)}{u^{2}} d u\right) \leq \\
\leq \frac{a(a+\eta(b, a))}{\eta(b, a)} \int_{a}^{a+\eta(b, a)} \frac{f(x)}{x^{2}} d x \leq \frac{f(a)+f(b)}{1-s}+\int_{0}^{1} d(a, a+\eta(b, a)) d t .
\end{array}
$$

Remark 2.4: (1) Corollary 1.3 will be reduced to Corollary 3.4 from [1, if we take $\eta(b, a)=b-a$.

(2) Corollary 1.3 will be reduced to Corollary 2.3 from [21], if $d(a, a+\eta(b, a))=0$. Moreover, if we take $\eta(b, a)=b-a$, we obtain Corollary 3.5 from [20].

Corollary 1.4. Let $f:[a, a+\eta(b, a)] \subseteq \mathbb{R} \backslash\{0\} \rightarrow \mathbb{R}$ be an integrable function. If $f$ is approximately harmonic tgs-preinvex function, and Condition $C$ holds, then

$$
\begin{gathered}
2\left(f\left(\frac{2 a(a+\eta(b, a))}{2 a+\eta(b, a)}\right)-\frac{a(a+\eta(b, a))}{\eta(b, a)} \frac{d\left(u,\left(\frac{1}{a}+\frac{1}{a+\eta(b, a)}-\frac{1}{u}\right)^{-1}\right)}{u^{2}} d u\right) \\
\leq \frac{a(a+\eta(b, a))}{\eta(b, a)} \int_{a}^{a+\eta(b, a)} \frac{f(x)}{x^{2}} d x \leq \frac{f(a)+f(b)}{6}+\int_{0}^{1} d(a, a+\eta(b, a)) d t .
\end{gathered}
$$

Moreover, if we take $\eta(b, a)=b-a$, we obtain the following Hermite-Hadamard inequality for approximately harmonic tgs-convex function

$$
\begin{aligned}
& 2\left(f\left(\frac{2 a b}{a+b}\right)-\frac{a b}{b-a} \int_{a}^{b} \frac{d\left(u,\left(\frac{1}{a}+\frac{1}{b}-\frac{1}{u}\right)^{-1}\right)}{u^{2}} d u\right) \\
\leq & \frac{a b}{b-a} \int_{a}^{b} \frac{f(x)}{x^{2}} d x \leq \frac{f(a)+f(b)}{6}+\int_{0}^{1} d(a, b) d t .
\end{aligned}
$$


If we take only $d(a, a+\eta(b, a))=0$, we get the following Hermite-Hadamard inequality for harmonic tgs-preinvex function

$$
2 f\left(\frac{2 a(a+\eta(b, a))}{2 a+\eta(b, a)}\right) \leq \frac{a(a+\eta(b, a))}{\eta(b, a)} \int_{a}^{a+\eta(b, a)} \frac{f(x)}{x^{2}} d x \leq \frac{f(a)+f(b)}{6} .
$$

Moreover, if we take $\eta(b, a)=b-a$, we obtain the following Hermite-Hadamard inequality for harmonic tgs-convex function

$$
2 f\left(\frac{2 a b}{a+b}\right) \leq \frac{a b}{b-a} \int_{a}^{b} \frac{f(x)}{x^{2}} d x \leq \frac{f(a)+f(b)}{6} .
$$

Corollary 1.5. Let $f:[a, a+\eta(b, a)] \subseteq \mathbb{R} \backslash\{0\} \rightarrow \mathbb{R}$ be an integrable function. If $f$ is approximately harmonic MT-preinvex function, and Condition $C$ holds, then

$$
\begin{gathered}
f\left(\frac{2 a(a+\eta(b, a))}{2 a+\eta(b, a)}\right)-\frac{a(a+\eta(b, a))}{\eta(b, a)} \int_{a}^{a+\eta(b, a)} \frac{d\left(u,\left(\frac{1}{a}+\frac{1}{a+\eta(b, a)}-\frac{1}{u}\right)^{-1}\right)}{u^{2}} d u \\
\leq \frac{a(a+\eta(b, a))}{\eta(b, a)} \int_{a}^{a+\eta(b, a)} \frac{f(x)}{x^{2}} d x \leq \frac{\pi}{4}(f(a)+f(b))+\int_{0}^{1} d(a, a+\eta(b, a)) d t .
\end{gathered}
$$

Moreover, if we take $\eta(b, a)=b-a$, we obtain the following Hermite-Hadamard inequality for approximately harmonic MT-convex function

$$
\begin{gathered}
f\left(\frac{2 a b}{a+b}\right)-\frac{a b}{b-a} \int_{a}^{b} \frac{d\left(u,\left(\frac{1}{a}+\frac{1}{b}-\frac{1}{u}\right)^{-1}\right)}{u^{2}} d u \\
\leq \frac{a b}{b-a} \int_{a}^{b} \frac{f(x)}{x^{2}} d x \leq \frac{\pi}{4}(f(a)+f(b))+\int_{0}^{1} d(a, b) d t .
\end{gathered}
$$

Additionally, if we take $d(a, a+\eta(b, a))=0$, we obtain

$$
f\left(\frac{2 a b}{a+b}\right) \leq \frac{a b}{b-a} \int_{a}^{b} \frac{f(x)}{x^{2}} d x \leq \frac{\pi}{4}(f(a)+f(b)) .
$$

Remark 2.5: Corollary 1.5 recapture Theorem 3.2 from [27, if we put $d(a, a+\eta(b, a))=0$. 
Corollary 1.6. Let $f:[a, a+\eta(b, a)] \subseteq \mathbb{R} \backslash\{0\} \rightarrow \mathbb{R}$ be an integrable function. If $f$ is approximately harmonic beta-preinvex function, and Condition $C$ holds, then

$$
\begin{aligned}
& 2^{(q+p)-1}\left(f\left(\frac{2 a(a+\eta(b, a))}{2 a+\eta(b, a)}\right)-\right. \\
& -\frac{a(a+\eta(b, a))}{\eta(b, a)} \int_{a}^{a+\eta(b, a) d\left(u,\left(\frac{1}{a}+\frac{1}{a+\eta(b, a)}-\frac{1}{u}\right)^{-1}\right)} \frac{u^{2}}{\eta(b, a)} \int_{a}^{a+\eta(b, a)} \frac{f(x)}{x^{2}} d x \leq \\
& \leq \frac{a(a+\eta(b, a))}{\leq(f(a)+f(b)) B(q+1, p+1)+\int_{0}^{1} d(a, a+\eta(b, a)) d t .}
\end{aligned}
$$

Moreover, if we take $\eta(b, a)=b-a$, we obtain the following Hermite-Hadamard inequality for approximately harmonic beta-convex function

$$
\begin{aligned}
& 2^{(q+p)-1}\left(f\left(\frac{2 a b}{a+b}\right)-\frac{a b}{b-a} \int_{a}^{b} \frac{d\left(u,\left(\frac{1}{a}+\frac{1}{b}-\frac{1}{u}\right)^{-1}\right)}{u^{2}} d u\right) \\
\leq & \frac{a b}{b-a} \int_{a}^{b} \frac{f(x)}{x^{2}} d x \leq(f(a)+f(b)) B(q+1, p+1)+\int_{0}^{1} d(a, b) d t .
\end{aligned}
$$

And if we take $d(a, a+\eta(b, a))=0$, we have the following Hermite-Hadamard inequality for harmonic beta-preinvex function

$$
\begin{aligned}
& 2^{(q+p)-1} f\left(\frac{2 a(a+\eta(b, a))}{2 a+\eta(b, a)}\right) \leq \frac{a(a+\eta(b, a))}{\eta(b, a)} \int_{a}^{a+\eta(b, a)} \frac{f(x)}{x^{2}} d x \leq \\
& \leq(f(a)+f(b)) B(q+1, p+1) .
\end{aligned}
$$

Moreover, if we choose $\eta(b, a)=b-a$, we get

$$
2^{(q+p)-1} f\left(\frac{2 a b}{a+b}\right) \leq \frac{a b}{b-a} \int_{a}^{b} \frac{f(x)}{x^{2}} d x \leq(f(a)+f(b)) B(q+1, p+1) .
$$


Lemma 1. Let $f: K=[a, a+\eta(b, a)] \subseteq \mathbb{R} \backslash\{0\} \rightarrow \mathbb{R}$ be a differentiable function on $I^{\circ}$ (interior of $\left.I\right)$. If $f^{\prime} \in L([a, a+\eta(b, a)])$ and $\lambda \in[0,1]$, then

$$
\begin{aligned}
\Upsilon_{f}(a, b ; \lambda)=\frac{a(a+\eta(b, a)) \eta(b, a)}{2} & \left(\int_{0}^{\frac{1}{2}} \frac{(\lambda-2 t)}{(a+t \eta(b, a))^{2}} f^{\prime}\left(\frac{a(a+\eta(b, a))}{a+t \eta(b, a)}\right) d t\right. \\
+ & \left.\int_{\frac{1}{2}}^{1} \frac{(2-\lambda-2 t)}{(a+t \eta(b, a))^{2}} f^{\prime}\left(\frac{a(a+\eta(b, a))}{a+t \eta(b, a)}\right) d t\right),
\end{aligned}
$$

where

$$
\begin{array}{r}
\Upsilon_{f}(a, b ; \lambda)=(1-\lambda) f\left(\frac{2 a(a+\eta(b, a))}{2 a+\eta(b, a)}\right)+\lambda \frac{f(a)+f(a+\eta(b, a))}{2}- \\
-\frac{a(a+\eta(b, a))}{\eta(b, a)} \int_{a}^{a+\eta(b, a)} \frac{f(x)}{x^{2}} d x .
\end{array}
$$

Proof. Let

$$
I_{1}=\frac{a(a+\eta(b, a)) \eta(b, a)}{2} \int_{0}^{\frac{1}{2}} \frac{(\lambda-2 t)}{(a+t \eta(b, a))^{2}} f^{\prime}\left(\frac{a(a+\eta(b, a))}{a+t \eta(b, a)}\right) d t
$$

and

$$
I_{2}=\frac{a(a+\eta(b, a)) \eta(b, a)}{2} \int_{\frac{1}{2}}^{1} \frac{(2-\lambda-2 t)}{(a+t \eta(b, a))^{2}} f^{\prime}\left(\frac{a(a+\eta(b, a))}{a+t \eta(b, a)}\right) d t
$$

Integrating by parts $I_{1}$, and then using the change of variable $\frac{a+t \eta(b, a)}{a(a+\eta(b, a))}=$ $\frac{1}{x}$, we get

$$
\begin{aligned}
I_{1}= & -\left.\frac{1}{2}(\lambda-2 t) f\left(\frac{a(a+\eta(b, a))}{a+t \eta(b, a)}\right)\right|_{0} ^{\frac{1}{2}}-\int_{0}^{\frac{1}{2}} f\left(\frac{a(a+\eta(b, a))}{a+t \eta(b, a)}\right) d t \\
= & \frac{1-\lambda}{2} f\left(\frac{2 a(a+\eta(b, a))}{2 a+\eta(b, a)}\right)+\frac{\lambda}{2} f(a+\eta(b, a)) \\
& -\frac{a(a+\eta(b, a))}{\eta(b, a)} \int_{\frac{2 a(a+\eta(b, a)}{2 a+\eta(b, a)}}^{a(x)} \frac{f(x)}{x^{2}} d x .
\end{aligned}
$$


Similarly, we obtain

$$
\begin{aligned}
& I_{2}=-\left.\frac{1}{2}(2-\lambda-2 t) f\left(\frac{a(a+\eta(b, a))}{a+t \eta(b, a)}\right)\right|_{t=\frac{1}{2}} ^{t=1}-\int_{\frac{1}{2}}^{1} f\left(\frac{a(a+\eta(b, a))}{a+t \eta(b, a)}\right) d t \\
& =\frac{1-\lambda}{2} f\left(\frac{2 a(a+\eta(b, a))}{2 a+\eta(b, a)}\right)+\frac{\lambda}{2} f(a)-\int_{\frac{1}{2}}^{1}\left(\frac{a(a+\eta(b, a))}{a+t \eta(b, a)}\right) d t \\
& =\frac{1-\lambda}{2} f\left(\frac{2 a(a+\eta(b, a))}{2 a+\eta(b, a)}\right)+\frac{\lambda}{2} f(a)-\frac{a(a+\eta(b, a))}{\eta(b, a)} \int_{a}^{2 a+\eta(b, a)} \\
& \frac{f(x)}{x^{2}} d t .
\end{aligned}
$$

Summing (2.6) and (2.7), we get the desired result.

Remark 2.6: Lemma 1 will be reduced to Lemma 6 from [10, if we choose $\eta(b, a)=b-a$ and to Lemma 2.5 from [9], if we take $\lambda=1$ and $\eta(b, a)=b-a$.

Theorem 2. Let $f:[a, a+\eta(b, a)] \subseteq \mathbb{R} \backslash\{0\} \rightarrow \mathbb{R}$ be a differentiable function on $(a, a+\eta(b, a))$ with $\eta(b, a)>0$ and $f^{\prime} \in L([a, a+\eta(b, a)])$. If $\left|f^{\prime}\right|^{q}$ is approximately harmonic h-preinvex function where $q>1$ with $\frac{1}{p}+\frac{1}{q}=1$, then

$$
\begin{aligned}
& \left|\Upsilon_{f}(a, b ; \lambda)\right| \\
\leq & \frac{a(a+\eta(b, a)) \eta(b, a)}{2}\left(\frac{\lambda^{p+1}+(1-\lambda)^{p+1}}{2(p+1)}\right)^{\frac{1}{p}} \\
& \times\left(\left(\mu_{1}(a, b)\left|f^{\prime}(a)\right|^{q}+\mu_{2}(a, b)\left|f^{\prime}(b)\right|^{q}+\mu_{3}(a, b)\right)^{\frac{1}{q}}\right. \\
& \left.+\left(\mu_{4}(a, b)\left|f^{\prime}(a)\right|^{q}+\mu_{5}(a, b)\left|f^{\prime}(b)\right|^{q}+\mu_{6}(a, b)\right)^{\frac{1}{q}}\right),
\end{aligned}
$$

where

$$
\begin{aligned}
\mu_{1}(a, b) & =\int_{0}^{\frac{1}{2}} \frac{h(t)}{(a+t \eta(b, a))^{2 q}} d t \\
\mu_{2}(a, b) & =\int_{0}^{\frac{1}{2}} \frac{h(1-t)}{(a+t \eta(b, a))^{2 q}} d t \\
\mu_{3}(a, b) & =\int_{0}^{\frac{1}{2}} \frac{d(a, a+\eta(b, a))}{(a+t \eta(b, a))^{2 q}} d t \\
\mu_{4}(a, b) & =\int_{\frac{1}{2}}^{1} \frac{h(t)}{(a+t \eta(b, a))^{2 q}} d t
\end{aligned}
$$




$$
\begin{aligned}
& \mu_{5}(a, b)=\int_{\frac{1}{2}}^{1} \frac{h(1-t)}{(a+t \eta(b, a))^{2 q}} d t \\
& \mu_{6}(a, b)=\int_{\frac{1}{2}}^{1} \frac{d(a, a+\eta(b, a))}{(a+t \eta(b, a))^{2 q}} d t
\end{aligned}
$$

and $\lambda \in[0,1]$.

Proof. From Lemma 1. properties of modulus and Hölder's inequality, we have

$$
\begin{aligned}
& \left|\Upsilon_{f}(a, b ; \lambda)\right| \\
\leq & \frac{a(a+\eta(b, a)) \eta(b, a)}{2} \\
& \times\left(\int_{0}^{\frac{1}{2}}|\lambda-2 t|^{p} d t\right)^{\frac{1}{p}}\left(\int_{0}^{\frac{1}{2}} \frac{1}{(a+t \eta(b, a))^{2 q}}\left|f^{\prime}\left(\frac{a(a+\eta(b, a))}{a+t \eta(b, a)}\right)\right|^{q} d t\right)^{\frac{1}{q}} \\
& \left.+\left(\int_{\frac{1}{2}}^{1}|2-\lambda-2 t|^{p} d t\right)^{\frac{1}{p}}\left(\int_{\frac{1}{2}}^{1} \frac{1}{(a+t \eta(b, a))^{2 q}}\left|f^{\prime}\left(\frac{a(a+\eta(b, a))}{a+t \eta(b, a)}\right)\right|^{q} d t\right)^{\frac{1}{q}}\right) \\
= & \frac{a(a+\eta(b, a)) \eta(b, a)}{2}\left(\frac{\lambda^{p+1}+(1-\lambda)^{p+1}}{2(p+1)}\right)^{\frac{1}{p}} \\
& \left.\left.\times\left(\int_{0}^{\frac{1}{2}} \frac{\left|f^{\prime}\left(\frac{a(a+\eta(b, a))}{a+t \eta(b, a)}\right)\right|^{q}}{(a+t \eta(b, a))^{2 q}} d t\right)^{\frac{1}{q}}+\int_{\frac{1}{2}}^{1} \frac{\left|f^{\prime}\left(\frac{a(a+\eta(b, a))}{a+t \eta(b, a)}\right)\right|^{q}}{(a+t \eta(b, a))^{2 q}} d t\right)^{\frac{1}{q}}\right) .
\end{aligned}
$$

Using the approximately harmonic $h$-preinvexity of $\left|f^{\prime}\right|^{q}$ on $[a, a+\eta(b, a)]$, we obtain

$$
\begin{aligned}
& \left|\Upsilon_{f}(a, b ; \lambda)\right| \leq \\
& \frac{a(a+\eta(b, a)) \eta(b, a)}{2}\left(\frac{\lambda^{p+1}+(1-\lambda)^{p+1}}{2(p+1)}\right)^{\frac{1}{p}}\left(\left.|| f^{\prime}(a)\right|^{q} \int_{0}^{\frac{1}{2}} \frac{h(t)}{(a+t \eta(b, a))^{2 q}} d t\right. \\
& \left.+\left|f^{\prime}(b)\right|^{q} \int_{0}^{\frac{1}{2}} \frac{h(1-t)}{(a+t \eta(b, a))^{2 q}} d t+\int_{0}^{\frac{1}{2}} \frac{d(a, a+\eta(b, a))}{(a+t \eta(b, a))^{2 q}} d t\right)^{\frac{1}{q}}
\end{aligned}
$$




$$
\begin{aligned}
& +\left(\left|f^{\prime}(a)\right|^{q} \int_{\frac{1}{2}}^{1} \frac{h(t)}{(a+t \eta(b, a))^{2 q}} d t\right. \\
& \left.\left.+\left|f^{\prime}(b)\right|^{q} \int_{\frac{1}{2}}^{1} \frac{h(1-t)}{(a+t \eta(b, a))^{2 q}} d t+\int_{\frac{1}{2}}^{1} \frac{d(a, a+\eta(b, a))}{(a+t \eta(b, a))^{2 q}} d t\right)^{\frac{1}{q}}\right) .
\end{aligned}
$$

Combining (2.7)-(2.13), we get the desired result.

Corollary 2.1. In Theorem 2 choosing $\lambda=1$, we obtain the following trapezium type inequality for approximately harmonic h-preinvex functions

$$
\begin{aligned}
& \left|\frac{f(a)+f(a+\eta(b, a))}{2}-\frac{a(a+\eta(b, a))}{\eta(b, a)} \int_{a}^{a+\eta(b, a)} \frac{f(x)}{x^{2}} d x\right| \\
& \leq \frac{a(a+\eta(b, a)) \eta(b, a)}{2^{1+\frac{1}{p}}}\left(\frac{1}{p+1}\right)^{\frac{1}{p}} \\
& \times\left(\left(\mu_{1}(a, b)\left|f^{\prime}(a)\right|^{q}+\mu_{2}(a, b)\left|f^{\prime}(b)\right|^{q}+\mu_{3}(a, b)\right)^{\frac{1}{q}}\right. \\
& \left.+\left(\mu_{4}(a, b)\left|f^{\prime}(a)\right|^{q}+\mu_{5}(a, b)\left|f^{\prime}(b)\right|^{q}+\mu_{6}(a, b)\right)^{\frac{1}{q}}\right) .
\end{aligned}
$$

Corollary 2.2. In Theorem 2 taking $\lambda=0$, we obtain the following midpoint type inequality for approximately harmonic h-preinvex functions

$$
\begin{aligned}
& \left|f\left(\frac{2 a(a+\eta(b, a))}{2 a+\eta(b, a)}\right)-\frac{a(a+\eta(b, a))}{\eta(b, a)} \int_{a}^{a+\eta(b, a)} \frac{f(x)}{x^{2}} d x\right| \\
\leq & \frac{a(a+\eta(b, a)) \eta(b, a)}{2^{1+\frac{1}{p}}}\left(\frac{1}{p+1}\right)^{\frac{1}{p}} \\
& \times\left(\left(\mu_{1}(a, b)\left|f^{\prime}(a)\right|^{q}+\mu_{2}(a, b)\left|f^{\prime}(b)\right|^{q}+\mu_{3}(a, b)\right)^{\frac{1}{q}}\right. \\
& \left.+\left(\mu_{4}(a, b)\left|f^{\prime}(a)\right|^{q}+\mu_{5}(a, b)\left|f^{\prime}(b)\right|^{q}+\mu_{6}(a, b)\right)^{\frac{1}{q}}\right) .
\end{aligned}
$$

Corollary 2.3. In Theorem 2 taking $\lambda=\frac{1}{3}$, we obtain the following Simpson type inequality for approximately harmonic h-preinvex functions

$$
\begin{aligned}
& \mid \frac{1}{6}\left(f(a)+4 f\left(\frac{2 a(a+\eta(b, a))}{2 a+\eta(b, a)}\right)+f(a+\eta(b, a))\right)- \\
& -\frac{a(a+\eta(b, a))}{\eta(b, a)} \int_{a}^{a+\eta(b, a)} \frac{f(x)}{x^{2}} d x \mid
\end{aligned}
$$




$$
\begin{aligned}
\leq & \frac{a(a+\eta(b, a)) \eta(b, a)}{6^{1+\frac{1}{p}}}\left(\frac{1+2^{p+1}}{p+1}\right) \\
& \times\left(\left(\mu_{1}(a, b)\left|f^{\prime}(a)\right|^{q}+\mu_{2}(a, b)\left|f^{\prime}(b)\right|^{q}+\mu_{3}(a, b)\right)^{\frac{1}{q}}\right. \\
& \left.+\left(\mu_{4}(a, b)\left|f^{\prime}(a)\right|^{q}+\mu_{5}(a, b)\left|f^{\prime}(b)\right|^{q}+\mu_{6}(a, b)\right)^{\frac{1}{q}}\right) .
\end{aligned}
$$

Theorem 3. Under the same conditions of Theorem 2, one has the following inequality

$$
\begin{aligned}
& \left|\Upsilon_{f}(a, b ; \lambda)\right| \\
\leq & \frac{a(a+\eta(b, a)) \eta(b, a)}{2}\left(\left(\vartheta_{1}(\lambda, a, b, p)\right)^{\frac{1}{p}}\right. \\
& \times\left(\left|f^{\prime}(a)\right|^{q} \int_{0}^{\frac{1}{2}} h(t) d t+\left|f^{\prime}(b)\right|^{q} \int_{0}^{\frac{1}{2}} h(1-t) d t+\int_{0}^{\frac{1}{2}} d(a, a+\eta(b, a)) d t\right)^{\frac{1}{q}} \\
& +\left(\vartheta_{2}(\lambda, a, b, p)\right)^{\frac{1}{p}} \\
& \left.\times\left(\left|f^{\prime}(a)\right|^{q} \int_{\frac{1}{2}}^{1} h(t) d t+\left|f^{\prime}(b)\right|^{q} \int_{\frac{1}{2}}^{1} h(1-t) d t+\int_{\frac{1}{2}}^{1} d(a, a+\eta(b, a)) d t\right)^{\frac{1}{q}}\right),
\end{aligned}
$$

where

$$
\begin{gathered}
\vartheta_{1}(\lambda, a, b, p)=\frac{\lambda^{p+1}}{2(p+1) a^{2 p}} \cdot 2 F_{1}\left(2 p, 1, p+2,-\lambda \frac{\eta(b, a)}{2 a}\right) \\
+\frac{(1-\lambda)^{p+1}}{2(p+1)\left(a+\frac{\lambda}{2} \eta(b, a)\right)^{2 p}} \cdot 2 F_{1}\left(2 p, p+1, p+2, \frac{(\lambda-1) \eta(b, a)}{2 a+\lambda \eta(b, a)}\right), \\
\vartheta_{2}(\lambda, a, b, p)=\frac{(1-\lambda)^{p+1}}{2(p+1)\left(a+\frac{1}{2} \eta(b, a)\right)^{2 p}} \cdot 2 F_{1}\left(2 p, 1, p+2, \frac{(\lambda-1) \eta(b, a)}{2 a+\eta(b, a)}\right) \\
+\frac{\lambda^{p+1}}{2(p+1)\left(a+\left(1-\frac{\lambda}{2}\right) \eta(b, a)\right)^{2 p}} \cdot 2 F_{1}\left(2 p, p+1, p+2,-\frac{\lambda \eta(b, a)}{2 a+(2-\lambda) \eta(b, a)}\right)
\end{gathered}
$$

and $\lambda \in[0,1]$.

Proof. From Lemma 1. properties of modulus and Hölder's inequality, we have

$$
\begin{aligned}
& \left|\Upsilon_{f}(a, b ; \lambda)\right| \\
\leq & \frac{a(a+\eta(b, a)) \eta(b, a)}{2} \\
& \times\left(\left(\int_{0}^{\frac{1}{2}} \frac{|\lambda-2 t|^{p}}{(a+t \eta(b, a))^{2 p}} d t\right)^{\frac{1}{p}}\left(\int_{0}^{\frac{1}{2}}\left|f^{\prime}\left(\frac{a(a+\eta(b, a))}{a+t \eta(b, a)}\right)\right|^{q} d t\right)^{\frac{1}{q}}\right.
\end{aligned}
$$




$$
\begin{aligned}
& \left.+\left(\int_{\frac{1}{2}}^{1} \frac{|2-\lambda-2 t|^{p}}{(a+t \eta(b, a))^{2 p}} d t\right)^{\frac{1}{p}}\left(\int_{\frac{1}{2}}^{1}\left|f^{\prime}\left(\frac{a(a+\eta(b, a))}{a+t \eta(b, a)}\right)\right|^{q} d t\right)^{\frac{1}{q}}\right) \\
& =\frac{a(a+\eta(b, a)) \eta(b, a)}{2} \\
& \times\left(\left(\vartheta_{1}(\lambda, a, b, p)\right)^{\frac{1}{p}}\left(\int_{0}^{\frac{1}{2}}\left|f^{\prime}\left(\frac{a(a+\eta(b, a))}{a+t \eta(b, a)}\right)\right|^{q} d t\right)^{\frac{1}{q}}\right. \\
& \left.+\left(\vartheta_{2}(\lambda, a, b, p)\right)^{\frac{1}{p}}\left(\int_{\frac{1}{2}}^{1}\left|f^{\prime}\left(\frac{a(a+\eta(b, a))}{a+t \eta(b, a)}\right)\right|^{q} d t\right)^{\frac{1}{q}}\right)
\end{aligned}
$$

Using the approximately harmonic $h$-preinvexity of $\left|f^{\prime}\right|^{q}$ on $[a, a+\eta(b, a)]$, we get

$$
\begin{aligned}
& \left|\Upsilon_{f}(a, b ; \lambda)\right| \\
\leq & \frac{a(a+\eta(b, a)) \eta(b, a)}{2}\left(\left(\vartheta_{1}(\lambda, a, b, p)\right)^{\frac{1}{p}}\right. \\
& \times\left(\left|f^{\prime}(a)\right|^{q} \int_{0}^{\frac{1}{2}} h(t) d t+\left|f^{\prime}(b)\right|^{q} \int_{0}^{\frac{1}{2}} h(1-t) d t+\int_{0}^{\frac{1}{2}} d(a, a+\eta(b, a)) d t\right)^{\frac{1}{q}} \\
& +\left(\vartheta_{2}(\lambda, a, b, p)\right)^{\frac{1}{p}} \\
& \left.\times\left(\left|f^{\prime}(a)\right|^{q} \int_{\frac{1}{2}}^{1} h(t) d t+\left|f^{\prime}(b)\right|^{q} \int_{\frac{1}{2}}^{1} h(1-t) d t+\int_{\frac{1}{2}}^{1} d(a, a+\eta(b, a)) d t\right)^{\frac{1}{q}}\right),
\end{aligned}
$$

where $\vartheta_{1}$ and $\vartheta_{2}$ are defined as in (2.14) and (2.15), respectively. The proof is completed.

Corollary 3.1. In Theorem 3 choosing $\lambda=1$, we obtain the following trapezium type inequality for approximately harmonic h-preinvex functions

$$
\begin{aligned}
& \left|\frac{f(a)+f(a+\eta(b, a))}{2}-\frac{a(a+\eta(b, a))}{\eta(b, a)} \int_{a}^{a+\eta(b, a)} \frac{f(x)}{x^{2}} d x\right| \\
\leq & \frac{a(a+\eta(b, a)) \eta(b, a)}{2^{1+\frac{1}{p}}(p+1)^{\frac{1}{p}}}\left(\frac{1}{a^{2}}\left({ }_{2} F_{1}\left(2 p, 1, p+2,-\frac{\eta(b, a)}{2 a}\right)\right)^{\frac{1}{p}}\right. \\
& \times\left(\left|f^{\prime}(a)\right|^{q} \int_{0}^{\frac{1}{2}} h(t) d t+\left|f^{\prime}(b)\right|^{q} \int_{0}^{\frac{1}{2}} h(1-t) d t+\int_{0}^{\frac{1}{2}} d(a, a+\eta(b, a)) d t\right)^{\frac{1}{q}}
\end{aligned}
$$




$$
\begin{aligned}
& +\frac{1}{\left(a+\frac{1}{2} \eta(b, a)\right)^{2}}\left({ }_{2} F_{1}\left(2 p, p+1, p+2,-\frac{\eta(b, a)}{2 a+\eta(b, a)}\right)\right)^{\frac{1}{p}} \\
& \left.\times\left(\left|f^{\prime}(a)\right|^{q} \int_{\frac{1}{2}}^{1} h(t) d t+\left|f^{\prime}(b)\right|^{q} \int_{\frac{1}{2}}^{1} h(1-t) d t+\int_{\frac{1}{2}}^{1} d(a, a+\eta(b, a)) d t\right)^{\frac{1}{q}}\right) .
\end{aligned}
$$

Corollary 3.2. In Theorem 3 taking $\lambda=0$, we obtain the following midpoint type inequality for approximately harmonic h-preinvex functions

$$
\begin{aligned}
& \left|f\left(\frac{2 a(a+\eta(b, a))}{2 a+\eta(b, a)}\right)-\frac{a(a+\eta(b, a))}{\eta(b, a)} \int_{a}^{a+\eta(b, a)} \frac{f(x)}{x^{2}} d x\right| \\
\leq & \frac{a(a+\eta(b, a)) \eta(b, a)}{2^{1+\frac{1}{p}}(p+1)^{\frac{1}{p}}}\left(\frac{1}{a^{2}}\left({ }_{2} F_{1}\left(2 p, p+1, p+2,-\frac{\eta(b, a)}{2 a}\right)\right)\right. \\
& \times\left(\left|f^{\prime}(a)\right|^{q} \int_{0}^{\frac{1}{2}} h(t) d t+\left|f^{\prime}(b)\right|^{q} \int_{0}^{\frac{1}{2}} h(1-t) d t+\int_{0}^{\frac{1}{2}} d(a, a+\eta(b, a)) d t\right)^{\frac{1}{q}} \\
& +\frac{\left(a+\frac{1}{2} \eta(b, a)\right)^{2}}{(1}\left({ }_{2} F_{1}\left(2 p, 1, p+2,-\frac{\eta(b, a)}{2 a+\eta(b, a)}\right)\right)^{\frac{1}{p}} \\
& \left.\times\left(\left|f^{\prime}(a)\right|^{q} \int_{\frac{1}{2}}^{1} h(t) d t+\left|f^{\prime}(b)\right|^{q} \int_{\frac{1}{2}}^{1} h(1-t) d t+\int_{\frac{1}{2}}^{1} d(a, a+\eta(b, a)) d t\right)^{\frac{1}{q}}\right) .
\end{aligned}
$$

Corollary 3.3. In Theorem 3 taking $\lambda=\frac{1}{3}$, we obtain the following Simpson type inequality for approximately harmonic h-preinvex functions

$$
\begin{aligned}
& \left|\frac{1}{6}\left(f(a)+4 f\left(\frac{2 a(a+\eta(b, a))}{2 a+\eta(b, a)}\right)+f(a+\eta(b, a))\right)-\frac{a(a+\eta(b, a))}{\eta(b, a)} \int_{a}^{a+\eta(b, a)} \frac{f(x)}{x^{2}} d x\right| \leq \\
& \leq \frac{a(a+\eta(b, a)) \eta(b, a)}{3^{1+\frac{1}{p}}(p+1)^{\frac{1}{p}}} \\
& \times\left(\left(\frac{{ }_{2} F_{1}\left(2 p, 1, p+2,-\frac{2 \eta(b, a)}{6 a+3 \eta(b, a)}\right)}{\left(a+\frac{1}{2} \eta(b, a)\right)^{2 p}}+\frac{{ }_{2} F_{1}\left(2 p, p+1, p+2,-\frac{\eta(b, a)}{6 a+5 \eta(b, a)}\right)}{\left(a+\frac{5}{6} \eta(b, a)\right)^{2 p}}\right)\right.
\end{aligned}
$$




$$
\begin{aligned}
& \times\left(\left|f^{\prime}(a)\right|^{q} \int_{0}^{\frac{1}{2}} h(t) d t+\left|f^{\prime}(b)\right|^{q} \int_{0}^{\frac{1}{2}} h(1-t) d t+\int_{0}^{\frac{1}{2}} d(a, a+\eta(b, a)) d t\right)^{\frac{1}{q}} \\
& +\left(\frac{{ }^{2} F_{1}\left(2 p, 1, p+2,-\frac{2 \eta(b, a)}{6 a+3 \eta(b, a)}\right)}{\left(a+\frac{1}{2} \eta(b, a)\right)^{2 p}}+\frac{{ }_{2} F_{1}\left(2 p, p+1, p+2,-\frac{\eta(b, a)}{6 a+5 \eta(b, a)}\right)}{\left(a+\frac{5}{6} \eta(b, a)\right)^{2}}\right)^{\frac{1}{p}} \\
& \left.\times\left(\left|f^{\prime}(a)\right|^{q} \int_{\frac{1}{2}}^{1} h(t) d t+\left|f^{\prime}(b)\right|^{q} \int_{\frac{1}{2}}^{1} h(1-t) d t+\int_{\frac{1}{2}}^{1} d(a, a+\eta(b, a)) d t\right)^{\frac{1}{q}}\right)
\end{aligned}
$$

Theorem 4. Let $f:[a, a+\eta(b, a)] \subseteq \mathbb{R} \backslash\{0\} \rightarrow \mathbb{R}$ be a differentiable function on $(a, a+\eta(b, a))$ with $\eta(b, a)>0$ and $f^{\prime} \in L([a, a+\eta(b, a)])$. If $\left|f^{\prime}\right|^{q}$ is approximately harmonic h-preinvex function where $q \geq 1$, then

$$
\begin{aligned}
& \left|\Upsilon_{f}(a, b ; \lambda)\right| \\
\leq & \frac{a(a+\eta(b, a)) \eta(b, a)}{2}\left(\left(\digamma_{1}(\lambda, a, b)\right)^{1-\frac{1}{q}}\right. \\
& \times\left(\iota_{1}(\lambda, a, b)\left|f^{\prime}(a)\right|^{q}+\imath_{2}(\lambda, a, b)\left|f^{\prime}(b)\right|^{q}+\imath_{3}(\lambda, a, b)\right)^{\frac{1}{q}} \\
& +\left(\digamma_{2}(\lambda, a, b)\right)^{1-\frac{1}{q}} \\
& \left.\times\left(\iota_{4}(\lambda, a, b)\left|f^{\prime}(a)\right|^{q}+\imath_{5}(\lambda, a, b)\left|f^{\prime}(b)\right|^{q}+\imath_{6}(\lambda, a, b)\right)^{\frac{1}{q}}\right)
\end{aligned}
$$

where

$$
\begin{gathered}
\digamma_{1}(\lambda, a, b)=\frac{\lambda^{2} \cdot_{2} F_{1}\left(2,1,3,-\frac{\lambda \eta(b, a)}{2 a}\right)}{4 a^{2}}+\frac{(1-\lambda)^{2} \cdot_{2} F_{1}\left(2,2,3, \frac{(\lambda-1) \eta(b, a)}{2 a+\lambda \eta(b, a)}\right)}{4\left(a+\frac{\lambda}{2} \eta(b, a)\right)^{2}}, \\
\digamma_{2}(\lambda, a, b)=\frac{(1-\lambda)^{2} \cdot{ }_{2} F_{1}\left(2,1,3,(\lambda-1) \frac{\eta(b, a)}{2 a+\eta(b, a)}\right)}{(2 a+\eta(b, a))^{2}} \\
\left.+\frac{\lambda^{2} \cdot_{2} F_{1}\left(2,2,3,-\frac{\lambda}{2} \eta(b, a)\right.}{\left(2 a+\left(1-\frac{\lambda}{2}\right) \eta(b, a)\right.}\right) \\
\end{gathered}
$$

and

$$
\begin{aligned}
& \imath_{1}(\lambda, a, b)=\int_{0}^{\frac{1}{2}} \frac{|\lambda-2 t| h(t)}{(a+t \eta(b, a))^{2}} d t, \\
& \imath_{2}(\lambda, a, b)=\int_{0}^{\frac{1}{2}} \frac{|\lambda-2 t| h(1-t)}{(a+t \eta(b, a))^{2}} d t,
\end{aligned}
$$




$$
\begin{gathered}
\imath_{3}(\lambda, a, b)=\int_{0}^{\frac{1}{2}} \frac{|\lambda-2 t| d(a, a+\eta(b, a))}{(a+t \eta(b, a))^{2}} d t, \\
\imath_{4}(\lambda, a, b)=\int_{\frac{1}{2}}^{1} \frac{|2-\lambda-2 t| h(t)}{(a+t \eta(b, a))^{2}} d t, \\
\imath_{5}(\lambda, a, b)=\int_{\frac{1}{2}}^{1} \frac{|2-\lambda-2 t| h(1-t)}{(a+t \eta(b, a))^{2}} d t, \\
\imath_{6}(\lambda, a, b)=\int_{\frac{1}{2}}^{1} \frac{|2-\lambda-2 t| d(a, a+\eta(b, a))}{(a+t \eta(b, a))^{2}} d t
\end{gathered}
$$

and $\lambda \in[0,1]$.

Proof. From Lemma 1 properties of modulus, and the well-known power mean inequality, we have

$$
\begin{aligned}
\leq & \frac{a(a+\eta(b, a)) \eta(b, a)}{2} \\
& \times\left(\Upsilon_{0}\left(\int_{0}^{\frac{1}{2}} \frac{|\lambda-2 t|}{(a+t \eta(b, a))^{2}} d t\right)^{1-\frac{1}{q}}\left(\int_{0}^{\frac{1}{2}} \frac{|\lambda-2 t|}{(a+t \eta(b, a))^{2}}\left|f^{\prime}\left(\frac{a(a+\eta(b, a))}{a+t \eta(b, a)}\right)\right|^{q} d t\right)^{\frac{1}{q}}\right. \\
& \left.+\left(\int_{\frac{1}{2}}^{1} \frac{|2-\lambda-2 t|}{(a+t \eta(b, a))^{2}} d t\right)^{1-\frac{1}{q}}\left(\int_{\frac{1}{2}}^{1} \frac{|2-\lambda-2 t|}{(a+t \eta(b, a))^{2}}\left|f^{\prime}\left(\frac{a(a+\eta(b, a))}{a+t \eta(b, a)}\right)\right|^{q} d t\right)^{\frac{1}{q}}\right) \\
= & \frac{a(a+\eta(b, a)) \eta(b, a)}{2} \\
& \times\left(\left(\digamma_{1}(\lambda, a, b)\right)^{1-\frac{1}{q}}\left(\int_{0}^{\frac{1}{2}} \frac{|\lambda-2 t|}{(a+t \eta(b, a))^{2}}\left|f^{\prime}\left(\frac{a(a+\eta(b, a))}{a+t \eta(b, a)}\right)\right|^{q} d t\right)^{\frac{1}{q}}\right. \\
& \left.+\left(\digamma_{2}(\lambda, a, b)\right)^{1-\frac{1}{q}}\left(\int_{\frac{1}{2}}^{1} \frac{|2-\lambda-2 t|}{(a+t \eta(b, a))^{2}}\left|f^{\prime}\left(\frac{a(a+\eta(b, a))}{a+t \eta(b, a)}\right)\right|^{q} d t\right)^{\frac{1}{q}}\right),
\end{aligned}
$$

where $\digamma_{1}$ and $\digamma_{2}$ are defined as in (2.16) and (2.17), respectively.

Now, using the approximately harmonic $h$-preinvexity of $\left|f^{\prime}\right|^{q}$ on $[a, a+\eta(b, a)]$, we obtain

$$
\begin{aligned}
& \left|\Upsilon_{f}(a, b ; \lambda)\right| \\
\leq & \frac{a(a+\eta(b, a)) \eta(b, a)}{2}\left(\left(\digamma_{1}(\lambda, a, b)\right)^{1-\frac{1}{q}}\right.
\end{aligned}
$$




$$
\begin{aligned}
& \times\left(\left|f^{\prime}(a)\right|^{q} \int_{0}^{\frac{1}{2}} \frac{|\lambda-2 t| h(t)}{(a+t \eta(b, a))^{2}} d t+\left|f^{\prime}(b)\right|^{q} \int_{0}^{\frac{1}{2}} \frac{|\lambda-2 t| h(1-t)}{(a+t \eta(b, a))^{2}} d t\right. \\
& \left.+\int_{0}^{\frac{1}{2}} \frac{|\lambda-2 t| d(a, a+\eta(b, a))}{(a+t \eta(b, a))^{2}} d t\right)^{\frac{1}{q}} \\
& +\left(\digamma_{2}(\lambda, a, b)\right)^{1-\frac{1}{q}}\left(\left|f^{\prime}(a)\right|^{q} \int_{\frac{1}{2}}^{1} \frac{|2-\lambda-2 t| h(t)}{(a+t \eta(b, a))^{2}} d t\right. \\
& \left.\left.\times\left|f^{\prime}(b)\right|^{q} \int_{\frac{1}{2}}^{1} \frac{|2-\lambda-2 t| h(1-t)}{(a+t \eta(b, a))^{2}} d t+\int_{\frac{1}{2}}^{1} \frac{|2-\lambda-2 t| d(a, a+\eta(b, a))}{(a+t \eta(b, a))^{2}} d t\right)^{\frac{1}{q}}\right)
\end{aligned}
$$

Using (2.16)- 2.23 into 2.24 , we obtain the desired result.

Corollary 4.1. In Theorem 4 choosing $\lambda=1$, we obtain the following trapezium type inequality for approximately harmonic h-preinvex functions

$$
\begin{aligned}
& \quad\left|\frac{f(a)+f(a+\eta(b, a))}{2}-\frac{a(a+\eta(b, a))}{\eta(b, a)} \int_{a}^{a+\eta(b, a)} \frac{f(x)}{x^{2}} d x\right| \\
& \leq \quad \frac{a(a+\eta(b, a)) \eta(b, a)}{2}\left(\left(\frac{1}{4 a^{2}} F_{1}\left(2,1,3,-\frac{\eta(b, a)}{2 a}\right)\right)^{1-\frac{1}{q}}\right. \\
& \quad \times\left(\breve{\imath}_{1}(a, b)\left|f^{\prime}(a)\right|^{q}+\breve{\imath}_{2}(a, b)\left|f^{\prime}(b)\right|^{q}+\breve{\imath}_{3}(a, b)\right)^{\frac{1}{q}} \\
& +\left(\frac{1}{(2 a+\eta(b, a))^{2}} F_{2}\left(2,2,3,-\frac{\eta(b, a)}{2 a+\eta(b, a)}\right)\right)^{1-\frac{1}{q}} \\
& \left.\quad \times\left(\breve{\imath}_{4}(a, b)\left|f^{\prime}(a)\right|^{q}+\breve{\iota}_{5}(a, b)\left|f^{\prime}(b)\right|^{q}+\breve{\imath}_{6}(a, b)\right)^{\frac{1}{q}}\right),
\end{aligned}
$$

where $\breve{\imath}_{i}(a, b)=\imath_{i}(1, a, b)$ with $\left(\imath_{i}(\lambda, a, b)\right)_{1 \leq i \leq 6}$ are defined as in (2.18)-2.23).

Corollary 4.2. In Theorem 4 taking $\lambda=0$, we obtain the following midpoint type inequality for approximately harmonic h-preinvex functions

$$
\begin{aligned}
& \left|f\left(\frac{2 a(a+\eta(b, a))}{2 a+\eta(b, a)}\right)-\frac{a(a+\eta(b, a))}{\eta(b, a)} \int_{a}^{a+\eta(b, a)} \frac{f(x)}{x^{2}} d x\right| \\
& \leq \frac{a(a+\eta(b, a)) \eta(b, a)}{2}\left(\left(\frac{1}{4 a^{2}} F_{2}\left(2,2,3,-\frac{\eta(b, a)}{2 a}\right)\right)^{1-\frac{1}{q}}\right. \\
& \quad \times\left(\widetilde{\imath}_{1}(a, b)\left|f^{\prime}(a)\right|^{q}+\widetilde{\imath}_{2}(a, b)\left|f^{\prime}(b)\right|^{q}+\widetilde{\imath}_{3}(a, b)\right)^{\frac{1}{q}}
\end{aligned}
$$




$$
\begin{aligned}
& +\left(\frac{1}{4(a+\eta(b, a))^{2}} F_{2}\left(2,2,3,-\frac{\eta(b, a)}{2 a+\eta(b, a)}\right)\right)^{1-\frac{1}{q}} \\
& \left.\times\left(\widetilde{\imath}_{4}(a, b)\left|f^{\prime}(a)\right|^{q}+\widetilde{\imath}_{5}(a, b)\left|f^{\prime}(b)\right|^{q}+\widetilde{\imath}_{6}(a, b)\right)^{\frac{1}{q}}\right),
\end{aligned}
$$

where $\widetilde{\imath}(a, b)=\imath_{i}(0, a, b)$ with $\left(\imath_{i}(\lambda, a, b)\right)_{1 \leq i \leq 6}$ are defined as in (2.18)-(2.23).

Corollary 4.3. In Theorem 4 taking $\lambda=\frac{1}{3}$, we obtain the following Simpson type inequality for approximately harmonic h-preinvex functions

$$
\begin{aligned}
& \left|\frac{1}{6}\left(f(a)+4 f\left(\frac{2 a(a+\eta(b, a))}{2 a+\eta(b, a)}\right)+f(a+\eta(b, a))\right)-\frac{a(a+\eta(b, a))}{\eta(b, a)} \int_{a}^{a+\eta(b, a)} \frac{f(x)}{x^{2}} d x\right| \\
\leq & \frac{a(a+\eta(b, a)) \eta(b, a)}{2}\left(\left(\frac{2 F_{1}\left(2,1,3,-\frac{\eta(b, a)}{6 a}\right)}{36 a^{2}}+\frac{{ }_{2} F_{1}\left(2,2,3,-\frac{2 \eta(b, a)}{(6 a+\eta(b, a))}\right)}{9\left(a+\frac{1}{6} \eta(b, a)\right)^{2}}\right){ }^{1-\frac{1}{q}}\right. \\
& \times\left(\hat{\imath}_{1}(a, b)\left|f^{\prime}(a)\right|^{q}+\hat{\imath}_{2}(a, b)\left|f^{\prime}(b)\right|^{q}+\hat{\imath}_{3}(a, b)\right)^{\frac{1}{q}} \\
& +\left(\frac{{ }_{2} F_{1}\left(2,1,3,-\frac{2 \eta(b, a)}{6 a+5 \eta(b, a)}\right)}{9(2 a+\eta(b, a))^{2}}+\frac{{ }_{2} F_{1}\left(2,2,3,-\frac{\eta(b, a)}{6 a+5 \eta(b, a)}\right)}{(6 a+5 \eta(b, a))^{2}}\right)^{1-\frac{1}{q}} \\
& \left.\times\left(\hat{\imath}_{4}(a, b)\left|f^{\prime}(a)\right|^{q}+\hat{\imath}_{5}(a, b)\left|f^{\prime}(b)\right|^{q}+\hat{\imath}_{6}(a, b)\right)^{\frac{1}{q}}\right),
\end{aligned}
$$

where $\hat{\imath}_{i}(a, b)=\imath_{i}\left(\frac{1}{3}, a, b\right)$ with $\left(\imath_{i}(\lambda, a, b)\right)_{1 \leq i \leq 6}$ are defined as in 2.18)-(2.23).

Corollary 4.4. In Theorem 4 if we take $q=1$, we obtain

$$
\begin{aligned}
& \left|\Upsilon_{f}(a, b ; \lambda)\right| \\
\leq & \frac{a(a+\eta(b, a)) \eta(b, a)}{2}\left(\left|f^{\prime}(a)\right| \int_{0}^{\frac{1}{2}}|\lambda-2 t|\left(\frac{h(t)}{(a+t \eta(b, a))^{2}}+\frac{h((1-t))}{(a+(1-t) \eta(b, a))^{2}}\right) d t\right. \\
& +\left|f^{\prime}(b)\right| \int_{0}^{\frac{1}{2}}|\lambda-2 t|\left(\frac{h(1-t)}{(a+t \eta(b, a))^{2}}+\frac{h(t)}{(a+(1-t) \eta(b, a))^{2}}\right) d t \\
& \left.+\int_{0}^{\frac{1}{2}}\left(\frac{|\lambda-2 t| d(a, a+\eta(b, a))}{(a+t \eta(b, a))^{2}}+\frac{|\lambda-2 t| d(a, a+\eta(b, a))}{(a+(1-t) \eta(b, a))^{2}}\right) d t\right) .
\end{aligned}
$$

Remark 2.7: From our Theorems 2, 3 and 4 and for the appropriate choices of functions $h(t)$ and $d(a, b)$, we can obtain new results for different types of preinvex functions as well as their analogues convex. We omit their proofs and the details are left to the interested readers.

\section{Conclusion}

This new class of functions called approximately harmonic $h$-preinvex can be applied to obtain several new results in convex analysis, related optimization theory, 
etc. The authors hope that these work may stimulate further research in different areas of pure and applied sciences.

Acknowledgements. We thank anonymous referee for his/her valuable suggestion regarding the manuscript.

\section{REFERENCES}

[1] M. U. Awan, M. A. Noor, M. V. Mihai, K. I. Noor, N. Akhtara, On Approximately Harmonic $h$-Convex Functions Depending on a Given Function, Filomat, 33 (12), (2019), 3783-3793.

[2] A. Barani, A. G. Ghazanfari, S. S. Dragomir, Hermite-Hadamard inequality for functions whose derivatives absolute values are preinvex, J. Inequal. Appl., 2012 (247), (2012), pp. 9.

[3] B. Bin-Mohsin, M. U. Awan, M. A. Noor, K. I. Noor, S. Iftikhar, A. G. Khan, Generalized beta-convex functions and integral inequalities, Inter. J. Anal. Appl., 14 (2), (2017), 180-192.

[4] S. S. Dragomir, Two mappings in connection to Hadamard's inequalities, J. Math. Anal. Appl., 167 (1), (1992), 49-56.

[5] S. S. Dragomir, R. P. Agarwal, Two new mappings associated with Hadamard's inequalities for convex functions, Appl. Math. Lett., 11 (3), (1998), 33-38.

[6] T. S. Du, J. G. Liao, Y. J. Li, Properties and integral inequalities of Hadamard-Simpson type for the generalized $(s, m)$-preinvex functions, J. Nonlinear Sci. Appl., 9 (5), (2016), $3112-3126$.

[7] J. Hadamard, Étude sur les propriétés des fonctions entières et en particulier ḋune fonction considérée par Riemann, J. Math. Pure. Appl., 58 (1893), 171-215.

[8] A. Iqbal, M. A. Khan, S. Ullah, Y. M. Chu, A. Kashuri, Hermite-Hadamard type inequalities pertaining conformable fractional integrals and their applications, AIP Advances, 8 (7), (2018), 1-18.

[9] İ. İşcan, Hermite-Hadamard type inequalities for harmonically convex functions, Hacet. J. Math. Stat., 43 (6), (2014), 935-942.

[10] İ. İşcan, Hermite-Hadamard and Simpson-like type inequalities for differentiable harmonically convex functions, J. Math., (2014), Art. ID 346305, pp. 10.

[11] A. Kashuri, R. Liko, Generalization of different type integral inequalities for generalized $(s, m)$-preinvex Godunova-Levin functions, J. Appl. Anal., 24 (2), (2018), 211-221.

[12] A. Kashuri, R. Liko, Some new Hermite-Hadamard type inequalities and their applications, Stud. Sci. Math. Hung., 56 (1), (2019), 103-142.

[13] A. Kashuri, E. Set, R. Liko, Some new fractional trapezium-type inequalities for preinvex functions, Fractal and Fractional, 3 (1), (2019), pp. 12.

[14] A. Kilbas, H. M. Srivastava, J. J. Trujillo, Theory and applications of fractional differential equations, North-Holland Mathematics Studies, 204. Elsevier Science B.V., Amsterdam, 2006.

[15] U. S. Kirmaci, Inequalities for differentiable mappings and applications to special means of real numbers and to midpoint formula, Appl. Math. Comput., 147 (1), (2004), 137-146.

[16] M. A. Khan, Y. M. Chu, A. Kashuri, R. Liko, G. Ali, Conformable fractional integrals versions of Hermite-Hadamard inequalities and their generalizations, J. Funct. Spaces, (2018), Art. ID 6928130, pp. 9.

[17] B. Meftah, M. Merad, A. Souahi, Some Hermite-Hadamard type inequalities for functions whose derivatives are quasi-convex, Jordan J. Math. Stat., 12 (2), (2019), 219-231.

[18] B. Meftah, M. Merad, N. Ouanas, A. Souahi, Some new Hermite-Hadamard type inequalities whose $n^{\text {th }}$ derivatives are convex, Acta Comment. Univ. Tartu. Math., 23 (2), (2019), 163178.

[19] S. R. Mohan, S. K. Neogy, On invex sets and preinvex functions, J. Math. Anal. Appl., 189 (3), (1995), 901-908. 
[20] M. A. Noor, K. I. Noor, M. U. Awan, S. Costache, Some integral inequalities for harmonically h-convex functions, Politehn. Univ. Bucharest Sci. Bull. Ser. A Appl. Math. Phys., 77 (1), (2015), 5-16.

[21] M. A. Noor, K. I. Noor, S. Iftikhar, Integral inequalities for differentiable relative harmonic preinvex functions (survey), TWMS J. Pure Appl. Math., 7 (1), (2016), 3-19.

[22] M. A. Noor, K. I. Noor, S. Iftikhar, Hermite-Hadamard inequalities for harmonic preinvex functions, Saussurea, 6 (1), (2016), 34-53.

[23] M. A. Noor, K. I. Noor, S. Iftikhar, K. Albany, Inequalities for MT-harmonic convex functions, J. Adv. Math. Stud., 9 (2), (2016), 194-207.

[24] M. A. Noor, K. I. Noor, S. Iftikhar, Fractional Ostrowski inequalities for harmonic hpreinvex functions, Facta Univ. Ser. Math. Inform., 31 (2), (2016), 417-445.

[25] M. A. Noor, K. I. Noor, S. Iftikhar, M. U. Awan, Strongly generalized harmonic convex functions and integral inequalities, J. Math. Anal., 7 (3), (2016), 66-77.

[26] M. A. Noor, K. I. Noor, S. Iftikhar, Hermite-Hadamard inequalities for strongly harmonic convex functions, J. Inequal. Spec. Funct., 7 (3), (2016), 99-113.

[27] M. A. Noor, K. I. Noor, S. Iftikhar, Harmonic MT-preinvex functions and integral inequalities, Rad Hrvat. Akad. Znan. Umjet. Mat. Znan., 20 (528), (2016), 51-70.

[28] M. A. Noor, K. I. Noor, S. Iftikhar, Harmonic beta-preinvex functions and inequalities, Inter. J. of Anal. Appl., 13 (2), (2017), 144-160.

[29] H. N. Shi, J. Zhang, Some new judgement theorems of Schur geometric and Schur harmonic convexities for a class of symmetric functions, J. Inequal. Appl., 2013 (527), (2013), pp. 9.

[30] Y. Zhang, T. S. Du, H. Wang, Y. J. Shen, A. Kashuri, Extensions of different type parameterized inequalities for generalized $(m, h)$-preinvex mappings via $k$-fractional integrals, J. Inequal. Appl., 49, (2018), pp. 30.

1 Laboratoire des télécommunications,

Faculté des Sciences et de la Technologie,

University of 8 May 1945 Guelma,

P.O. Box 401, 24000 Guelma, Algeria

Email address: badrimeftah@yahoo.fr

2 Department of Mathematics, Faculty of Technical Science, University Ismail Qemali, Vlora, Albania

Email address: artionkashuri@gmail.com

Received: 27.1 .2020

Revised: 28.3 .2020

Accepted: 30.3 .2020 